\title{
Supporting Information for Deciphering Flupyrimin Binding Surface on the Insect Nicotinic Acetylcholine Receptor
}

Takehito Terajima, ${ }^{1}$ Tomonori Suzuki, ${ }^{2}$ Ryo Horikoshi, ${ }^{3}$ Shohei Doi, ${ }^{1}$ Mizuki Nakamura, ${ }^{1}$ Fumika Kobayashi, ${ }^{1}$ Kathleen A. Durkin, ${ }^{4}$ Kenji Shimomura, ${ }^{1}$ Satoshi Nakamura, ${ }^{3}$ Kazumi Yamamoto, ${ }^{3}$ and Motohiro Tomizawa ${ }^{1, *}$

${ }^{1}$ Departments of Chemistry and ${ }^{2}$ Molecular Microbiology, Faculty of Life Sciences, Tokyo University of Agriculture; ${ }^{3}$ Agricultural and Veterinary Research Division, Meiji Seika Pharma Co. Ltd.; ${ }^{4}$ College of Chemistry, University of California, Berkeley

*To whom correspondence should be addressed: mt204882@nodai.ac.jp

Table S1. Acaricidal activity of FLP against the various ticks via topical application onto the animal skin

\begin{tabular}{|c|c|c|c|c|c|c|c|}
\hline & species & $\begin{array}{l}\text { treated } \\
\text { animal }\end{array}$ & dose & stage & replication & $\begin{array}{c}\text { observation } \\
\text { (day after treatment) }\end{array}$ & $\%$ control \\
\hline Brown dog tick & Rhipicephalus sanguineus & dog & 25 mg/kg & adult & 4 & 29 & 99 \\
\hline Castor bean tick & Ixodes ricinus & dog & $25 \mathrm{mg} / \mathrm{kg}$ & adult & 4 & 30 & 82 \\
\hline Asian longhorned tick & Haemaphysalis longicornis & dog & 20 mg/kg & larva & 3 & 30 & 100 \\
\hline
\end{tabular}

The formulation containing FLP was treated onto the target animals via topical application, and then the target ticks were infested onto the skin of the target animals at the determined timing before observation. The number of remaining on the animals or detached from them was counted and \% control was calculated following the formula.

$\%$ Control $=[$ (the remaining number of ticks in the untreated check) - (the remaining number of ticks in the treated animals $) /($ the remaining number of ticks in the untreated check)] 100. 
The remaining number of ticks indicates the counted number or the number as below.

(the remaining number of ticks) $=$ (the number of ticks infested)-(the number of ticks detached from the animals). 